\title{
Receipt of Telepsychiatry and Emergency Department Visit Outcomes in New York State
}

\author{
Cordelia Zhong ${ }^{1} \cdot$ Rain E. Freeman ${ }^{2} \cdot$ Krislyn M. Boggs ${ }^{1} \cdot$ Kori S. Zachrison ${ }^{1}$. \\ Jingya Gao ${ }^{1}$. Janice A. Espinola ${ }^{1} \cdot$ Carlos A. Camargo Jr ${ }^{1}$ iD
}

Accepted: 4 January 2021 / Published online: 15 February 2021

(c) The Author(s), under exclusive licence to Springer Science+Business Media, LLC part of Springer Nature 2021

\begin{abstract}
Telepsychiatry has made psychiatric care more accessible to emergency department (ED) patients. To date, most telepsychiatry studies have focused on specific populations or small groups of EDs. This study sought to examine the potential role of telepsychiatry across a wider range of EDs by comparing visit dispositions for psychiatric visits in EDs that did (versus did not) receive telepsychiatry services. ED telepsychiatry service status was identified from the 2016 National ED Inventory-USA and then linked to psychiatric visits from the 2016 New York State Emergency Department Databases/State Inpatient Databases. Unadjusted analyses and multivariable logistic regression models were used to evaluate associations between an ED's telepsychiatry service status and two clinical outcomes: use of observation services and ED visit disposition. Across all psychiatric ED visits, 712,236 were in EDs without telepsychiatry while 101,025 were in EDs with telepsychiatry. Most (99.8\%) visits were in urban EDs. In multivariable logistic regression models, psychiatric visits in EDs with telepsychiatry services had lower odds (adjusted odds ratio 0.30) of using observation services compared to visits in EDs without telepsychiatry. The receipt of ED telepsychiatry is associated with lower usage of observation services for psychiatric visits, likely reducing the amount of time spent in the ED and mitigating the ongoing problem of ED crowding. An overwhelming majority of visits in EDs with telepsychiatry services were in urban hospitals with existing psychiatric services. Factors affecting the delivery and effectiveness of telepsychiatry services to hospitals lacking in psychiatric resources merit further investigation.
\end{abstract}

Keywords Psychiatry · Telemedicine - Emergency department - Observation services · Visit disposition

Carlos A. Camargo Jr

ccamargo@partners.org

1 Department of Emergency Medicine, Massachusetts General Hospital, 125 Nashua St, Suite 920, Boston, MA 02114-1101, USA

2 Center for Population Health Research, University of Montana, Missoula, MT, USA 


\section{Introduction}

The prevalence of mental illness has increased in the U.S. since the early 1990s [1]. In 2016, over 55 million adults were living with a mental illness or substance use disorder [2]. Emergency departments (EDs) are increasingly bearing the brunt of these patients, with the rate of ED visits related to mental illness or substance use disorder increasing by $44.1 \%$ between 2006 and 2014 [3]. With many EDs lacking in the ability to efficiently care for psychiatric patients [4], these visits are more likely than non-psychiatric visits to result in inpatient admission [2]. Yet, even with increased need for resources, the number of inpatient psychiatric beds has not kept up with demand, leaving psychiatric patients spending hours in the ED waiting for transfer to an open bed and creating crowding in the ED [5]. Previous efforts to solve ED crowding has focused on diverting psychiatric patients out of the ED. In recent years, many have argued instead for the creation of an effective environment within the ED for the emergency management and treatment of psychiatric patients, with an increasing interest in the ability of telepsychiatry to alleviate the rising demand [4].

Telepsychiatry, or the receipt of telehealth services for the evaluation of ED patients who require mental or behavioral health care [6], is one of the fastest growing segments of telehealth [7]. In 2016, $20 \%$ of US EDs received telepsychiatry services [6]. The use of telepsychiatry in EDs allows remote access to psychiatric providers with expertise in emergency psychiatry, helps improve patient care, facilitate patient discharge, and prevent ED crowding [4]. Preliminary studies suggest that, in comparison to traditional face-to-face delivery of mental health services, telepsychiatry is comparable in both effectiveness and patient satisfaction $[8,9]$. Patients who did not receive ED telepsychiatry services appeared to spend more time waiting for treatment or an inpatient bed, were more likely to have their visit result in inpatient admission, and were less likely to attend outpatient follow-ups [10-12]. Despite the promise of telepsychiatry, much of the current research on ED telepsychiatry use focuses either on specific patient populations based on age [9, 12] or only includes hospital-based EDs enrolled into a specific telepsychiatry intervention program $[10,11]$. We sought to investigate the role of telepsychiatry across a wider and more representative range of EDs. Thus, we examined all ED visits across New York State to investigate the difference between psychiatric visits in EDs that did not receive telepsychiatry services versus visits in EDs that did.

Because disposition of patients with psychiatric complaints in the ED continues to be an area of national focus, we focused our investigation on the use of observation services during the ED visit [13-15] and the final ED visit disposition [13, 14]. While many studies have observed the effect of telepsychiatry on ED length of stay and admission/transfer outcomes in psychiatric patients [10-12], we were also interested in the observation services variable. Observation services are used when patients require a period of treatment or monitoring before an admission or discharge decision can be made, with most cases of observation stays lasting between 8 to 48 hours [15]. While under observation, a psychiatric patient would not only still be taking up space in the ED, but also be subjected to chaotic ED environments, which have been shown to worsen symptoms in psychiatric patients [4]. Thus we hoped to explore the ability of telepsychiatry to reduce need for observation services and help facilitate more efficient discharges. We hypothesized that relative to visits in EDs with telepsychiatry services, visits in EDs that did not receive telepsychiatry services would be more likely to use observation services and have higher rates of admission. 


\section{Methods}

\section{Study Design}

This observational study was based on data gathered from an institutional-level survey of U.S. EDs, which was then linked to a state database with patient outcomes. New York was chosen due to the fact that the state had rural and urban EDs as well as teaching hospitals and Critical Access Hospitals (CAH). The study was approved by the Human Research Committee of Partners Healthcare.

\section{National Emergency Department Inventory}

Using the National Emergency Department Inventory (NEDI)-USA database [16], we identified 194 New York EDs open in 2016. EDs were included in NEDI-USA if they were open 24 hours per day, 7 days per week, year-round and were available for use by the general public. This included freestanding EDs (EDs that are not physically connected to a hospital) [17]. We mailed a one-page survey to all ED directors up to three times and then contacted nonresponding EDs to complete the survey through telephone interview. The 2016 NEDI-USA survey asked ED directors to report basic characteristics of their ED, including annual visit volumes and telemedicine use (see Online Supplement).

\section{Exposure Measurement}

Receipt of ED telepsychiatry was assessed with the question: "Does your ED receive telemedicine for patient evaluation?" [18]. Those who responded yes were then asked to report the clinical applications for which they used telemedicine. EDs whose directors reported use of telemedicine for psychiatry or wrote-in that they used telemedicine for "behavioral health" or "mental health" were classified as receiving telepsychiatry. We also collected: rural location, defined as ED location outside of a core-based statistical area (CBSA) [19]; CAH [20] designation; and Council of Teaching Hospitals (COTH) [21] designation.

\section{Outcome Variables}

EDs identified in the 2016 NEDI-USA database were linked to the 2016 American Hospital Association (AHA) database [22] using methods previously described [23]. Using AHA ID numbers, the NEDI-USA EDs were then linked to the New York 2016 State Emergency Department Databases (SEDD) and New York 2016 State Inpatient Database (SID). Visits in EDs that did not respond to the 2016 NEDI-USA survey or to EDs unable to be linked to the 2016 AHA database were excluded. We first compiled an overview of psychiatric services available to New York hospitals in 2016, comparing hospitals with EDs that did not report receipt of telepsychiatry services versus hospitals with EDs that did. Through the use of SEDD/SID ICD-10-CM diagnosis codes [24], we then identified visits that received a psychiatric-related primary, secondary, or tertiary diagnosis in SEDD and psychiatric visits with subsequent admissions by admitting diagnosis in SID. We obtained patient characteristics, including age, sex, race/ethnicity, 
insurance status, and median household income (based on patient's ZIP code) [13, 14]. We then identified the use of observation services during the visit and the final disposition of the ED visit. The visit dispositions of interest were transfer to a short-term hospital, transfer to another type of facility (including psychiatric hospitals), or admission through the ED.

\section{Statistical Analysis}

Descriptive statistics are presented as both frequencies and percentages. We used chisquare test to evaluate bivariate associations between an ED's telepsychiatry service status and other characteristics at visit and hospital levels. A two-sided $\mathrm{P}<0.05$ was considered statistically significant. We then used both unadjusted and multivariable logistic regression models to examine the associations between telepsychiatry status and two outcomes: use of observation services and final ED visit disposition, with a random intercept for hospital to account for variation in dependent variables across hospitals. In the disposition model, outcome was defined as visits admitted through ED or transferred to a short-term hospital or other type of facility. Non-case was defined as visits with routine discharge. Other types of facility include a skilled nursing facility, intermediate care facility (ICF), and a psychiatric hospital or psychiatric unit. All multivariable models were adjusted for several visit and hospital characteristics. In sensitivity analyses, we re-ran our multivariable models in subpopulations restricted to adults only and patients with a primary diagnosis in SEDD. Patient data from SID had to be excluded from the primary diagnosis population, as it was not possible to tell whether the psychiatric diagnosis in the ED was primary or secondary. Results are reported as odds ratios (ORs) with $95 \%$ confidence intervals (95\% CIs). All analyses were performed with SAS software, version 9.4 (SAS; Cary, NC).

\section{Results}

In 2016, there were 194 EDs open in New York State. The data from these EDs were consolidated into 160 observations [23] (each observation representing between 1 and 3 New York EDs) linked across the NEDI-USA, AHA, and SEDD/SID databases, with 18 (11\%) of those being EDs that received telepsychiatry. After excluding visits in EDs that did not respond to the 2016 NEDI-USA survey, visits in the remaining 133 EDs then formed our analytic sample (Fig. 1).

\section{Psychiatric Services in New York State}

The EDs within our analytic sample were first examined for hospital psychiatric services based on data from the AHA database (Table 1). Eighteen hospitals reported having no psychiatric services. Of the 18 EDs that reported receiving telepsychiatry services in 2016 , 8 (44\%) also had psychiatric beds within their hospital, 13 (72\%) had psychiatric emergency services, and 8 (44\%) had psychiatric outpatient services. Only 1 was a CAH, with 3 others identified as teaching hospitals. EDs without telepsychiatry saw a median of 37,000 annual total ED visits, with an interquartile range of 16,000 - 75,000 visits. ED's with 


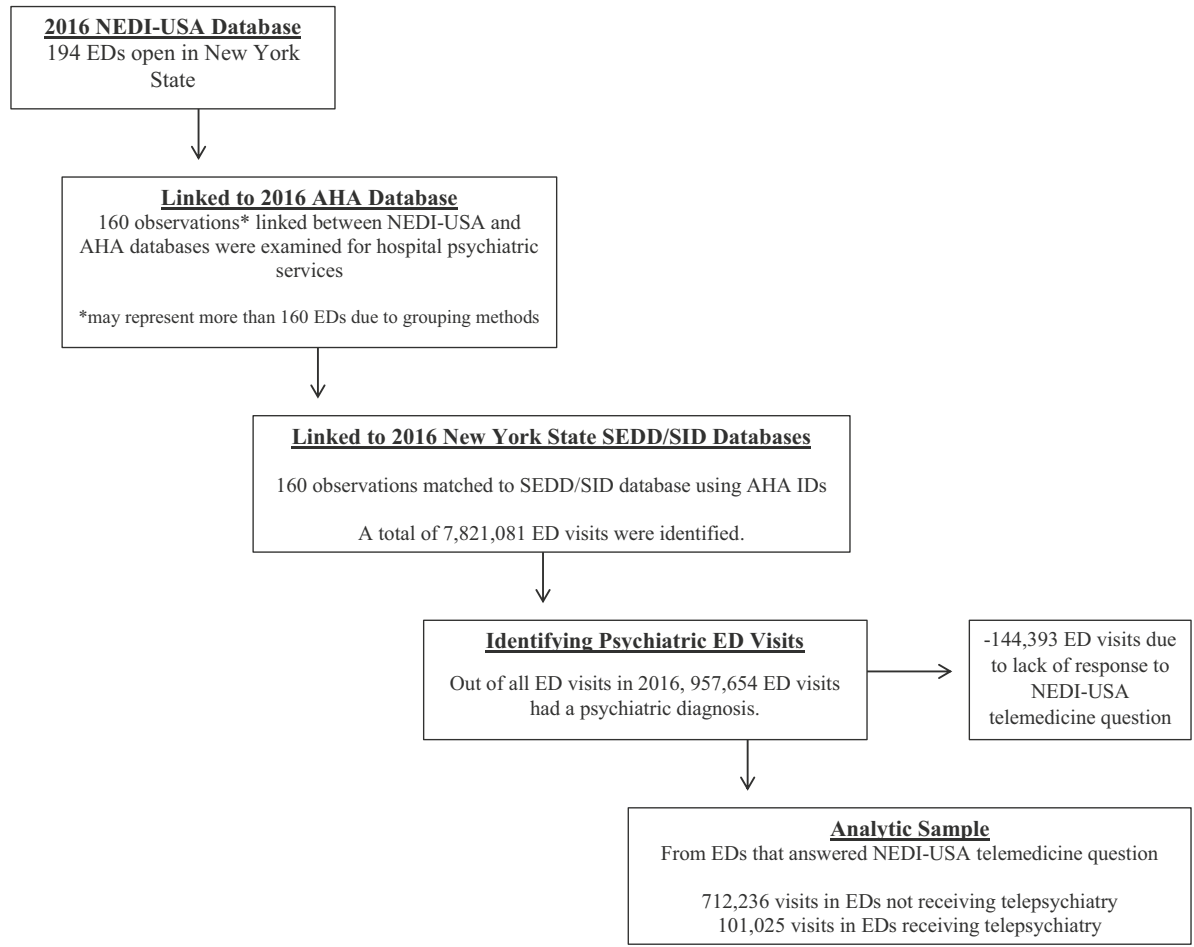

Fig. 1 Inclusion flow diagram. Abbreviations: NEDI, National Emergency Department Inventory; ED, Emergency Department; AHA, American Hospital Association; SEDD, State Emergency Department Databases; SID, State Inpatient Database

telepsychiatry services saw a median of 50,000 total ED visits per year, with an interquartile range of 34,500-87,500 visits.

\section{Psychiatric ED Visits and Telepsychiatry}

Across all EDs consolidated into our analytic sample, there were 7,821,081 total ED visits. Among those visits, 957,654 (12\%) received a psychiatric diagnosis. Among these visits, $712,236(74 \%)$ were in an ED without telepsychiatry and $101,025(11 \%)$ were in an ED with telepsychiatry; 144,393 (15\%) visits were excluded due to their unknown telepsychiatry status. The receipt of telepsychiatry services was associated with several patient and hospital characteristics (Table 2). In bivariate comparisons, visits in EDs with telepsychiatry services were more likely to occur in an urban ED (99.8\% vs 98\%), EDs with >40,000 annual visits ( $83 \%$ vs $77 \%$ ), and among patients with public (63\% vs $62 \%$ ) or private insurance ( $24 \%$ vs $21 \%)$.

\section{Telepsychiatry Receipt Status and Visit Disposition}

Observation services were used in $2.3 \%$ of visits presenting to EDs with telepsychiatry, versus $2.7 \%$ of patients presenting to EDs without. We found that visits in EDs without 
Table 1 Facility characteristics of New York State Emergency Departments without and with telepsychiatry services, 2016

\begin{tabular}{|c|c|c|c|}
\hline Hospital Characteristics & $\begin{array}{l}\text { EDs answered tel- } \\
\text { emedicine question } \\
(n=133)\end{array}$ & $\begin{array}{l}\text { EDs without } \\
\text { telepsychiatry } \\
\text { services } \\
(\mathrm{n}=115)\end{array}$ & $\begin{array}{l}\text { EDs with } \\
\text { telepsychiatry } \\
\text { services } \\
(\mathrm{n}=18)\end{array}$ \\
\hline \multicolumn{4}{|l|}{ Psychiatric Services, n(\%) } \\
\hline Psychiatric care beds & $60(45)$ & $52(45)$ & $8(44)$ \\
\hline $\begin{array}{l}\text { Alcoholism-chemical dependency care } \\
\text { services }\end{array}$ & $33(25)$ & $30(26)$ & $3(17)$ \\
\hline Psychiatric education services & $51(38)$ & $41(36)$ & $10(56)$ \\
\hline Psychiatric emergency services & $69(52)$ & $56(49)$ & $13(72)$ \\
\hline Psychiatric outpatient services & $59(44)$ & $51(44)$ & $8(44)$ \\
\hline Psychiatric partial hospitalization services & $21(16)$ & $18(16)$ & $3(17)$ \\
\hline Psychiatric residential treatment & $7(5.2)$ & $6(5.2)$ & $1(5.6)$ \\
\hline None & $18(14)$ & $15(13)$ & $3(17)$ \\
\hline \multicolumn{4}{|l|}{ Annual total ED visits, $\mathrm{n}(\%)$} \\
\hline$<10,000$ & $14(11)$ & $13(11)$ & $1(5.6)$ \\
\hline $10,000-19,999$ & $20(15)$ & $19(17)$ & $1(5.6)$ \\
\hline $20,000-39,999$ & $32(24)$ & $27(23)$ & $5(28)$ \\
\hline$\geq 40,000$ & $67(50)$ & $56(49)$ & $11(61)$ \\
\hline Teaching Hospital, $\mathrm{n}(\%)$ & $13(10)$ & $10(8.7)$ & $3(17)$ \\
\hline Critical Access Hospital, n(\%) & $16(12)$ & $15(13)$ & $1(5.6)$ \\
\hline \multicolumn{4}{|l|}{ Location of Hospital (CBSA code), $n(\%)$} \\
\hline Urban & $122(92)$ & $105(91)$ & $17(94)$ \\
\hline Rural & $11(8.3)$ & $10(8.7)$ & $1(5.6)$ \\
\hline
\end{tabular}

Abbreviations: ED emergency department, $C B S A$ core-based statistical area

telepsychiatry were less likely to result in admission or transfer than visits in EDs with telepsychiatry. When comparing psychiatric visits in EDs without telepsychiatry versus those with telepsychiatry, $12 \%$ versus $14 \%$ resulted in admission or transfer to a shortterm hospital, $83 \%$ versus $78 \%$ resulted in routine discharge, and $2 \%$ versus $5 \%$ resulted in transfer to another type of facility.

In multivariable analyses (Table 3), psychiatric visits in EDs with telepsychiatry were three times less likely to use observation services $(\mathrm{P}=0.03)$, after adjusting for patient demographic characteristics and hospital characteristics. In a separate multivariable analysis for final ED visit disposition (Table 4), psychiatric visits in EDs without telepsychiatry had similar odds of admission through the ED, transfer to a short-term hospital, or transfer to another type of facility, compared to psychiatric visits in EDs with telepsychiatry. In a sensitivity analysis restricting our analytical population to those with documentation of a primary psychiatric diagnosis in SEDD, visits in EDs with telepsychiatry remained less likely to have used observation services (adjusted OR 0.35, 95\% CI 0.12-0.99, $\mathrm{P}=0.048$ ), and ED telepsychiatry status remained unassociated with ED disposition. In another sensitivity analysis restricting our analytical population to adults only, observation service also occurred less often in visits in EDs with telepsychiatry service (adjusted OR 0.30 , 95\% CI 0.10-0.85, $\mathrm{P}=0.02$ ). Adult patients had similar ED dispositions regardless of ED telepsychiatry status. 


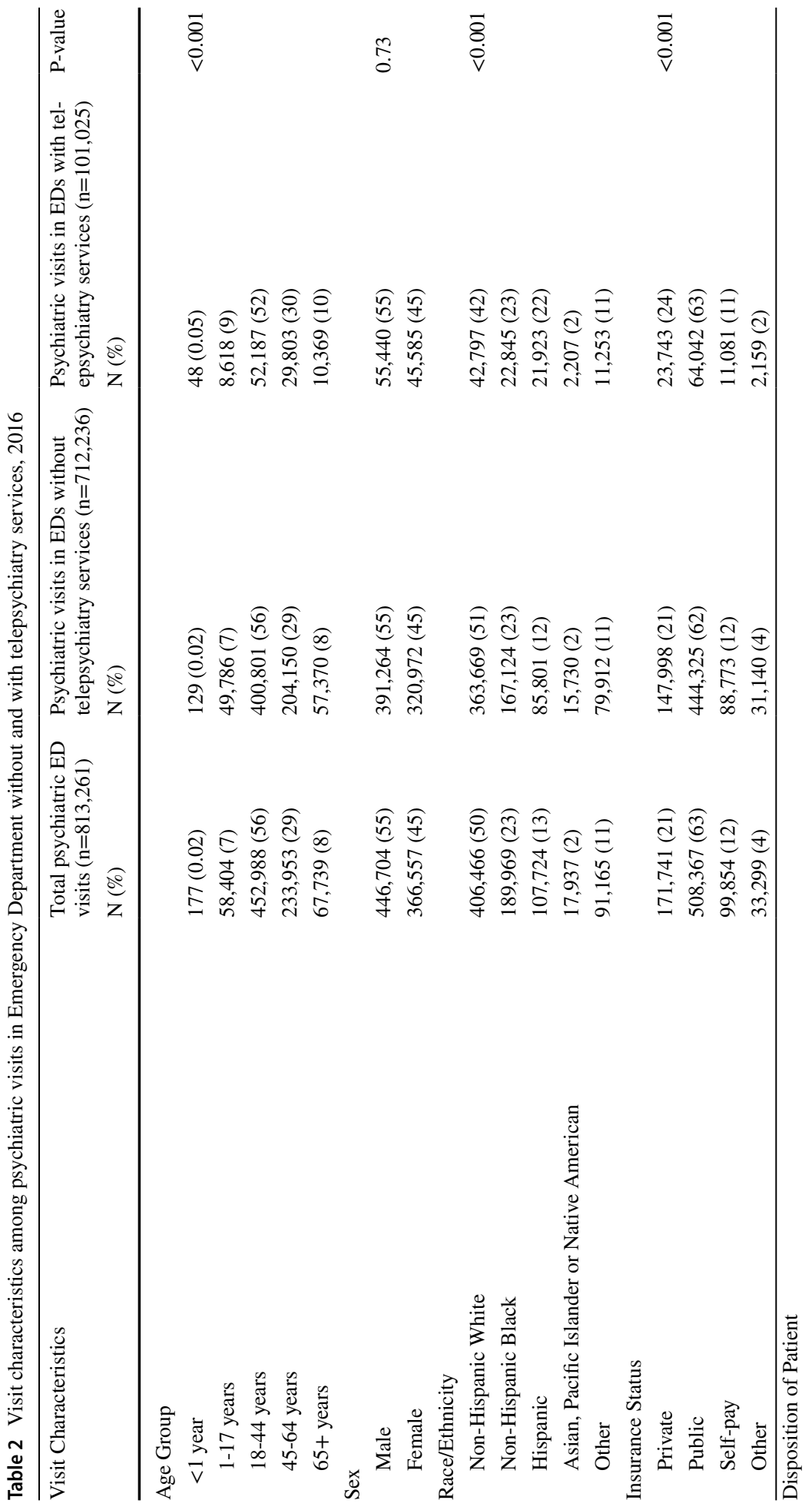




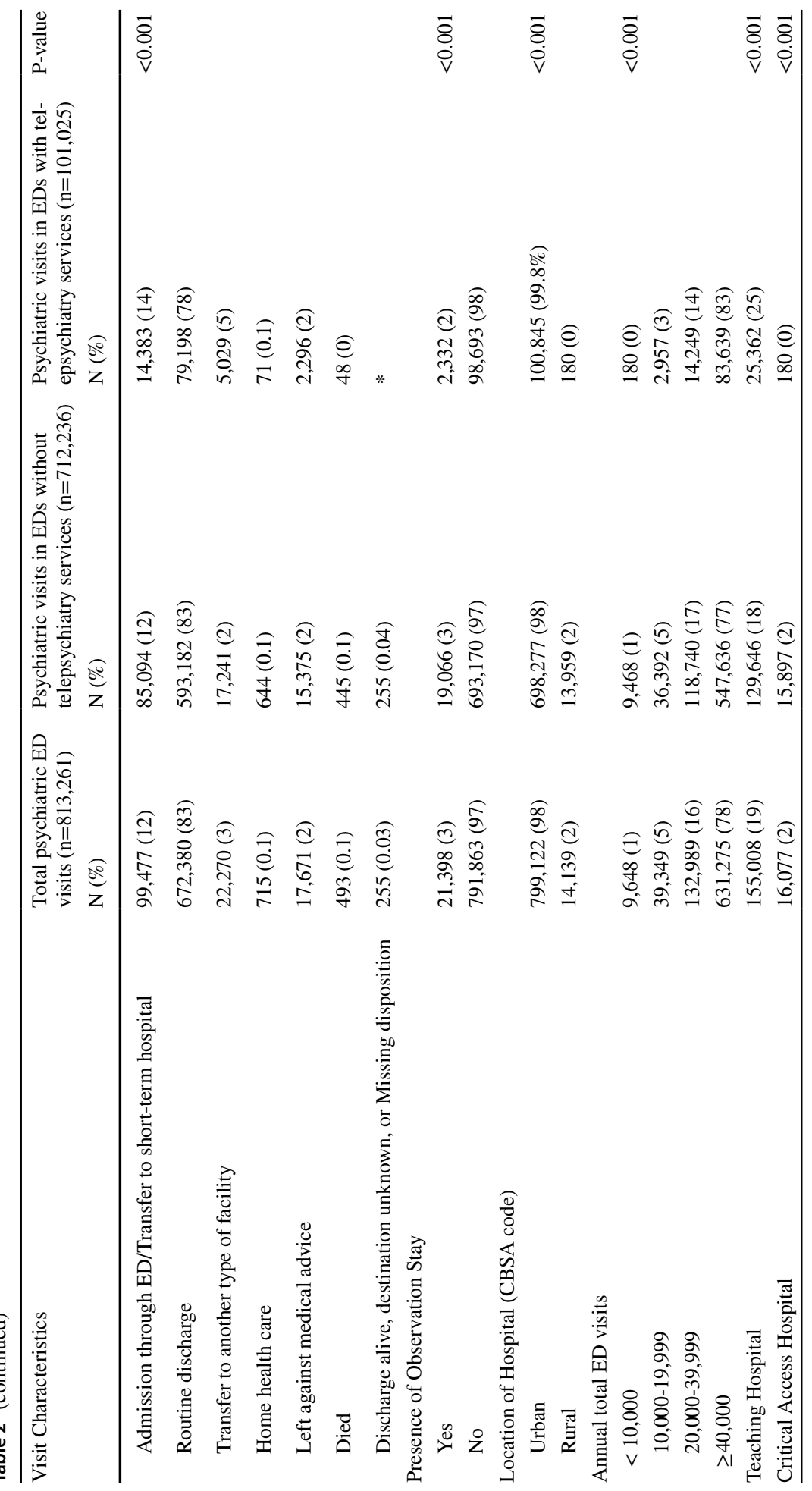




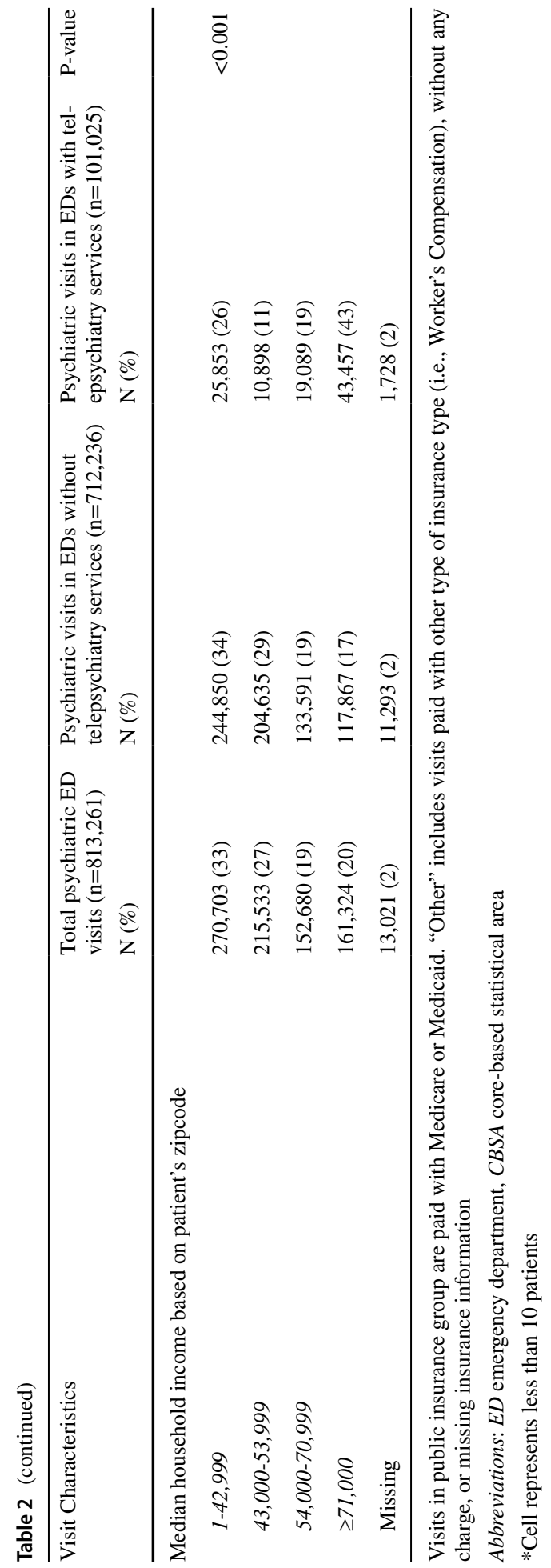




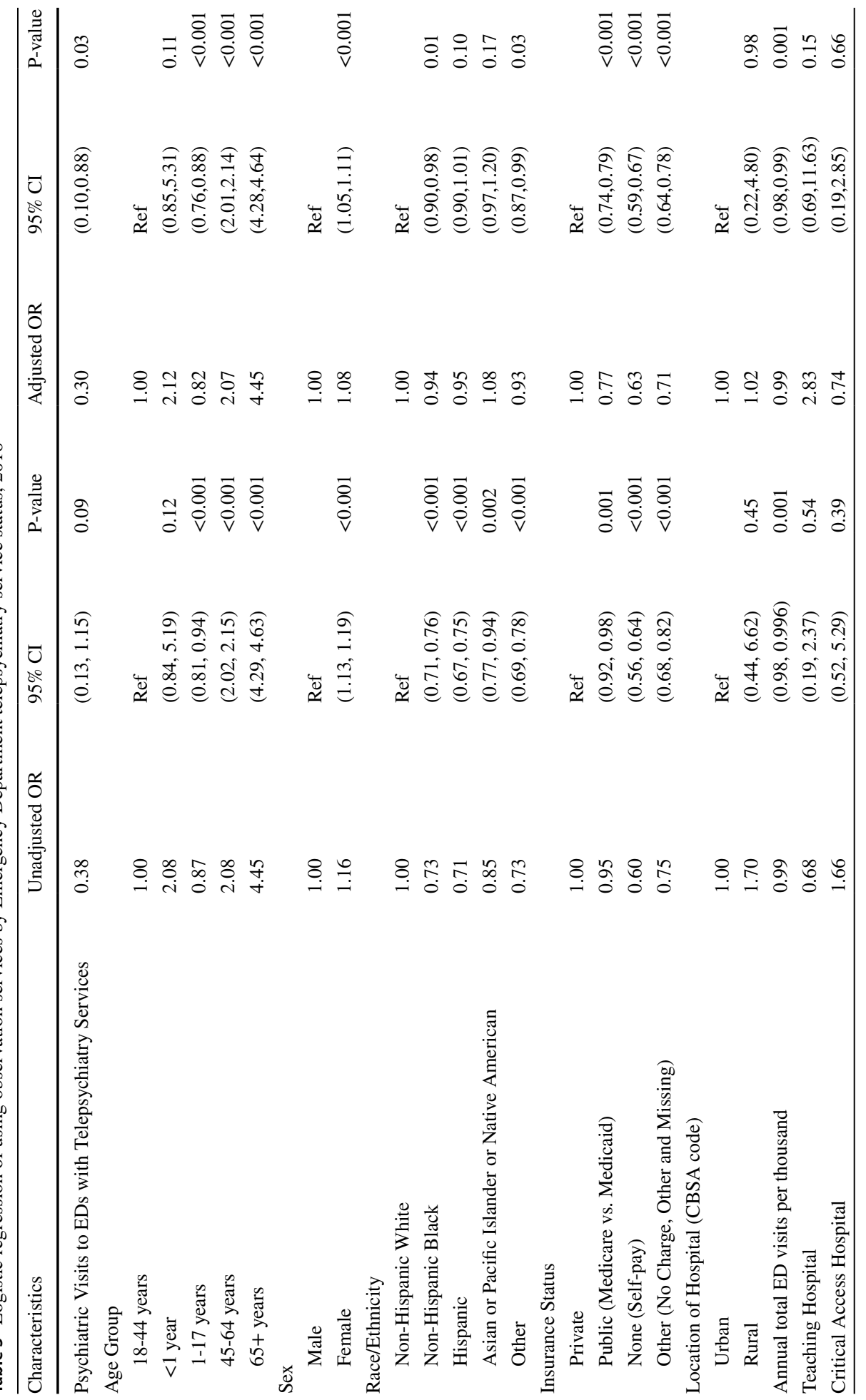




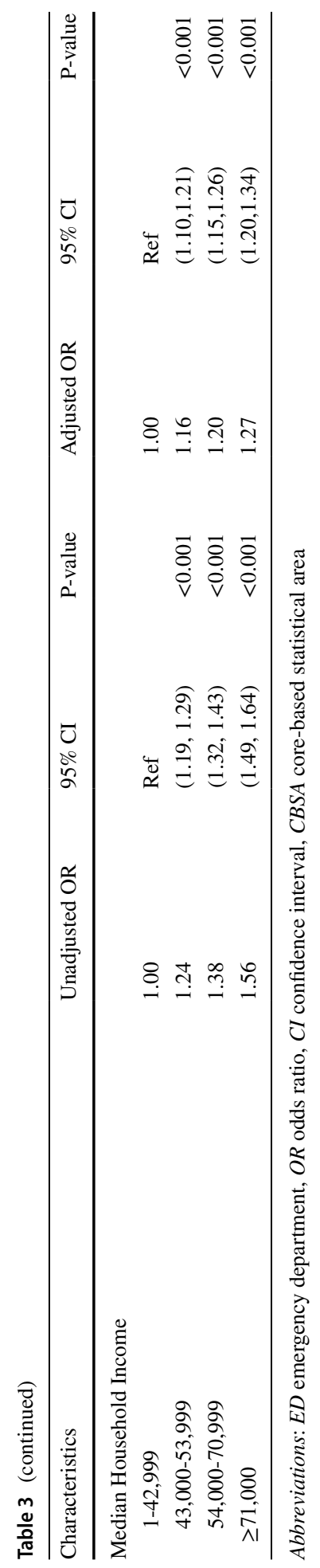




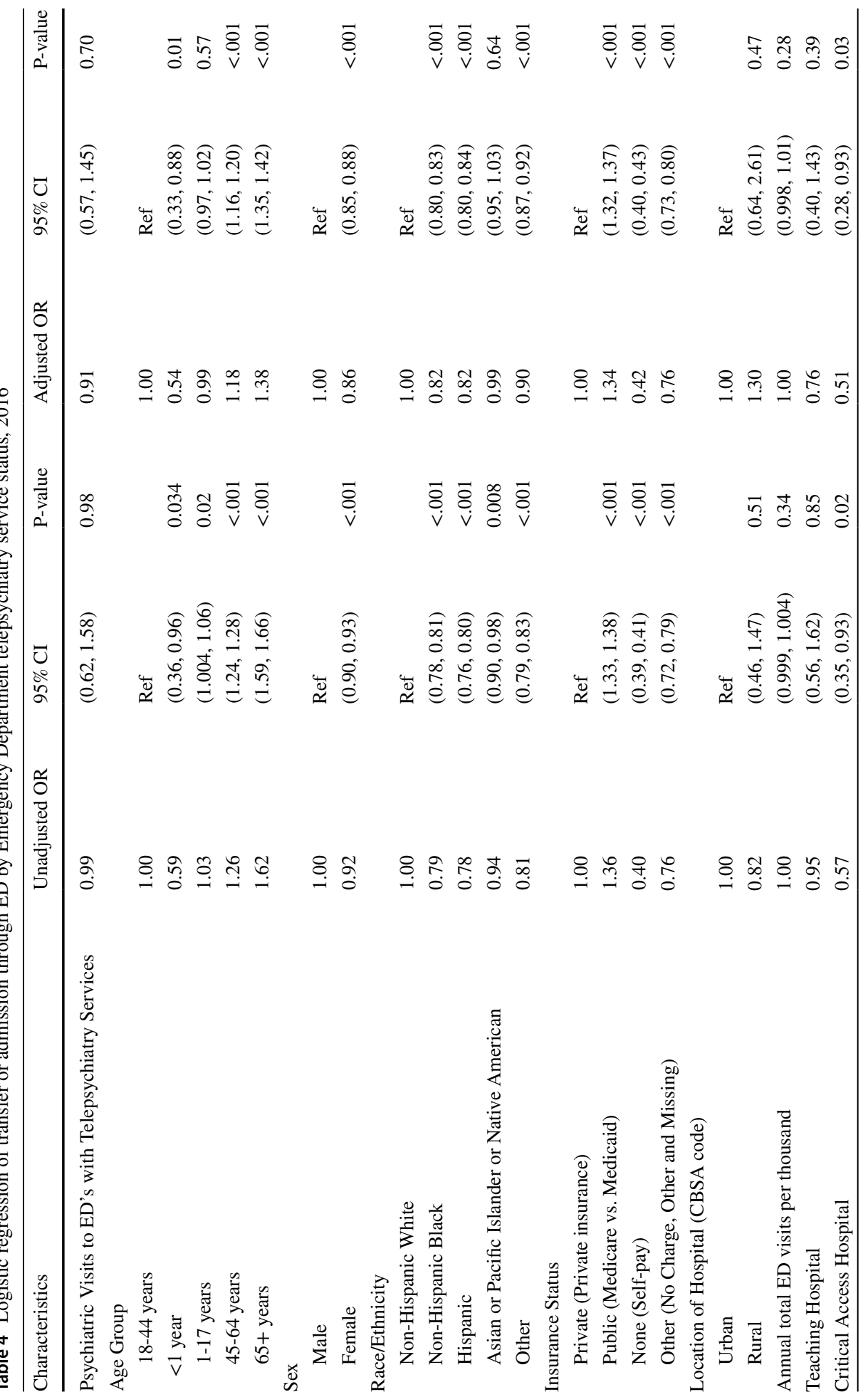




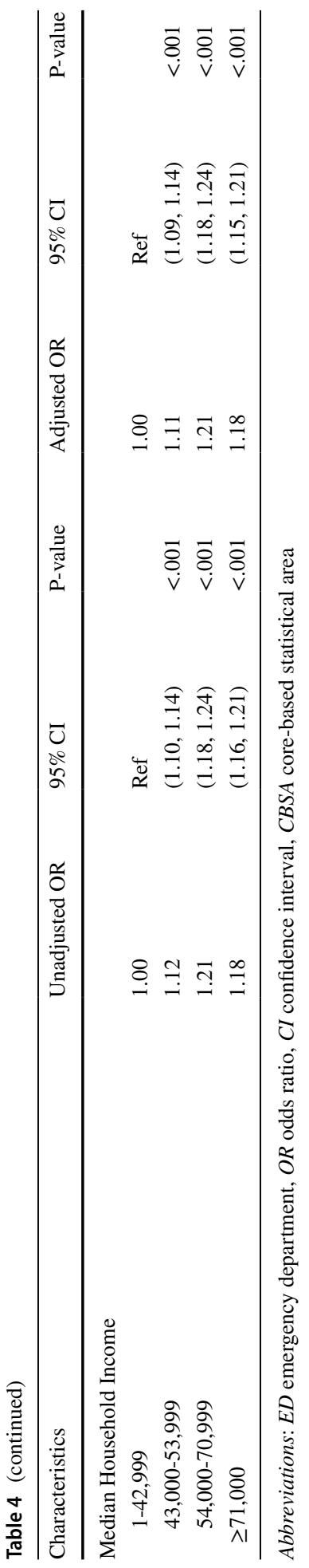




\section{Discussion}

Through linkage of multiple 2016 datasets, we were able to compare visit- and hospitallevel characteristics across 813,261 visits in New York EDs, with an analytic focus on the ED's receipt of telepsychiatry services. We examined the association of telepsychiatry in the ED with two specific outcomes of an ED visit; the use of observation services and final ED visit disposition. Psychiatric visits in EDs without telepsychiatry, compared to those with, were three times more likely to have used observation services, but still had similar odds of being admitted or transferred as their final ED disposition.

To our knowledge, this study is the first to investigate the role of telepsychiatry across an entire state's EDs, with a focus on patient disposition. Prior studies on the potential of telepsychiatry for use within ED settings were conducted within very specific settings, with some only focusing on pediatric populations or only among certain hospital systems [9-12]. While one prior study investigated the impact of telepsychiatry across South Carolina, only visits in hospitals enrolled in the state telepsychiatry program were eligible to be matched to visits in hospitals without telepsychiatry, limiting the generalizability to hospitals not receiving telepsychiatry through this dedicated state program [10]. By examining all EDs across New York State, we found that visits in EDs with telepsychiatry had lower odds of observation unit use while maintaining similar admission and transfer outcomes as visits in EDs without. Though we conducted a separate analysis excluding children from the population, we did not find that it materially affected results. This finding is consistent with previous research, in which, telepsychiatry has been suggested as a potential solution for patients in EDs without in-house psychiatric services, with evidence suggesting that telepsychiatry is comparable on several measures [8-12].

With many ED's lacking the ability to care for psychiatric patients, these patients are often left in the ED waiting for transfer to an inpatient bed, exacerbating the problem of crowding in EDs [4]. Research suggests that the use of specialized observation services can reduce inpatient admission rates for psychiatric patients. However, it must be noted that this requires investment into the specialized resources that would allow psychiatric patients under observation to receive efficient and safe care [25], something most EDs lack [4]. Even with an efficient environment, the use of observation services, by definition, increases the amount of time spent in the ED [15] and does not resolve the problem of crowding in the ED, especially when other ED patients may require observation. In our findings, visits in EDs with telepsychiatry were less likely to use observation services while maintaining similar disposition outcomes. Though a low percentage of all psychiatric ED visits used observation services (Table 2), it is possible that what is considered use of observation services in one ED may be considered part of ED length of stay in another ED with no official observation unit [15]. Our findings still lend support to telepsychiatry's ability to reduce overall time spent in EDs [11], whether the visit involves official use of observation services or non-classified time spent waiting for treatment.

With the increase in psychiatric-related visits in the ED in recent years, rural EDs have struggled to accommodate these patients as an increase in urban migration has led to decreased access to specialists and consultants in rural areas [26]. In New York, 40 out of its 62 counties were designated as Mental Health Shortage areas in 2014 [27]. Telepsychiatry has been touted before as a solution for rural EDs to access necessary mental health resources [28]. Despite this, we found that among our analytic sample, $98 \%$ of visits were in EDs in urban hospitals, with $99.8 \%$ of visits in EDs with telepsychiatry services occurring in urban hospitals. Only one hospital with telepsychiatry services reported having a 
$\mathrm{CAH}$ designation. These findings differ from results of a nationally representative sample, where EDs in rural areas or carrying CAH designations made up a higher proportion of EDs that received telepsychiatry [6]. It is unclear why a state with a documented need for mental health resources in its rural counties [29] sees the highest rate of telepsychiatry use in its urban hospitals or in hospitals with in-house psychiatric services. However, based on recent research on barriers to use of telemedicine by rural hospitals [26], we can hypothesize that cost and technologic concerns have played a role in the lack of telepsychiatry services in rural EDs despite research supporting its use.

The financial sustainability of telepsychiatry programs is not a new concern, as many programs are grant-funded and risk dissolution upon the disappearance of the grant [30]. In regards to technology, hospitals often use different electronic medical records systems, making it difficult to transfer information gathered by the ED provider to the off-site clinician [31]. To address these concerns, we encourage more research into alternative telepsychiatry delivery methods that help to reduce cost and technological difficulties. For example, a recent study examined the effect of psychiatric assessment officers in rural New York EDs [32]. A group of officers, including licensed social workers, mental health counselors, and psychiatric nurses, were integrated into EDs for 8 hours daily to help perform psychiatric evaluations and coordinate further care via telepsychiatry. This method cut costs by reducing the amount of time a licensed psychiatrist was needed and making use of lowercost behavioral health providers.

In addition to the cost of telepsychiatry itself, hospitals must also obtain reimbursement for these patients through their insurance companies, an issue that telehealth experts have identified as a major barrier for incorporating telemedicine into everyday healthcare practice [33]. Within the US, Medicare covers telepsychiatry services only under certain specifications (e.g., historically for patients living in rural areas) [34, 35]. However, while it was found that there was an increase in rural Medicare beneficiaries who were able to access telepsychiatry services from 2004 to 2014, an overwhelming amount were using telepsychiatry to supplement in-person psychiatric care. Only $15 \%$ of beneficiaries used telepsychiatry as their sole source of mental health care [36]. Within the psychiatric ED visits that we examined, most (63\%) were paid for by public insurance (including Medicare) and 98\% occurred within urban settings. These findings parallel those found by Mehrotra et al. [36] and further support the benefit of Medicare coverage pivoting from its historic targeted population of rural beneficiaries. By adding the ability to cover telepsychiatry use in urban EDs, this could ensure the program is truly helping to increase access to care for those who do not have other sources of emergency psychiatric care. Currently, due to the COVID-19 pandemic, restrictions have been relaxed to allow Medicare coverage for use of telehealth in urban areas [37]. An extension of the current waiver could help motivate hospitals previously unwilling to invest in telepsychiatry services due to their location in a non-rural area.

\section{Limitations}

Our study has potential limitations. First, our decision to focus on one state limits the study's statistical power as compared to analyzing a national sample. However, we chose New York not only due to its many Mental Health Shortage areas [27] but also because New York has both rural and urban EDs (Table 1), in addition to teaching hospitals and CAHs $[19,20]$. The state had 7.8 million ED visits in 2016, which gave us a relatively large sample to analyze. Additionally, psychiatric patients also often struggle with physical 
health issues, which can complicate care and cause situations where their psychiatric illness is not the primary diagnosis for their ED visit but is still an underlying issue that requires treatment [38]. These could have excluded them from being in our visit population, however, we mitigated this issue by including visits where the secondary and tertiary diagnosis in SEDD were also psychiatric related. Our sensitivity analysis also showed the same difference in observation service usage and similar patient's disposition among primarily diagnosed visits in EDs with and without telepsychiatry. In addition, the data we used was primarily administrative data for billing purposes and does not offer the in-depth detail of individual chart reviews. However, the SEDD/SID databases are often used to study patient-level data and outcomes across an entire state [39, 40]. Lastly, our study identified telepsychiatry service status as an ED-level variable rather than a visit-level variable, thus it is unknown which individual patients received telepsychiatry services versus in-person exams. Given that this is the first study to examine ED telepsychiatry state wide, review of service status at the facility level is appropriate. Given our finding that visits in EDs with telepsychiatry are less likely to use observation services, we encourage future studies to look into the possibility of identifying telepsychiatry use on a visit-level.

\section{Conclusion}

We investigated the association of ED-based telepsychiatry and ED visit outcomes across all psychiatric-related ED visits in New York State during 2016. Compared to EDs without telepsychiatry, the presence of telepsychiatry was associated with decreased odds of an observation stay, with similar final ED disposition. Factors affecting the delivery and effectiveness of telepsychiatry services to ED patients merit further investigation, especially in EDs with few resources or with a lack of any emergency psychiatric services whatsoever.

Supplementary Information The online version contains supplementary material available at https://doi. org/10.1007/s11126-021-09886-y.

Acknowledgements The authors thank Ashley Sullivan, MS MPH, for her scientific contributions and leadership of the EMNet Coordinating Center and Maranatha Teferi for her work on the linkage of NEDI-USA, AHA, SEDD/SID databases. We also thank EMNet clinical research coordinators and interns for helping with data collection.

Authors' Contributions $\mathrm{CZ}, \mathrm{KMB}$, and CAC conceived and designed the study. KMB and CAC obtained funding, and supervised the conduct of the study. KMB and JAE managed the data. CZ, JG, and JAE designed the data analysis and JG completed analyses. All authors contributed to interpretation of the results. $\mathrm{CZ}$ drafted the article and all authors contributed substantially to its revision.

Funding This study was supported, in part, by a grant from the R Baby Foundation (New York, NY). The authors had complete freedom to direct the analysis and its reporting, without any influence from the sponsor.

Data Availability The authors confirm that the data supporting the findings of this study are available within the article.

Code Availability Not applicable.

\section{Declarations}

Ethics Approval This article does not contain any studies with human participants or animals performed by any of the authors. 
Consent to Participate Not applicable.

Consent for Publication Not applicable.

Conflict of Interest None of the authors have any potential conflicts of interest that affected the reporting of this work.

\section{References}

1. Larkin GL, Claassen CA, Emond JA, Pelletier AJ, Camargo CA. Trends in U.S. Emergency Department visits for mental health conditions, 1992 to 2001. Psychiatr Serv. 2005;56(6):671-7.

2. Owens PL, Fingar KR, McDermott KW, Muhuri PK, Heslin KC. Inpatient stays involving mental and substance use disorders, 2016: statistical brief \#249. Rockville: Agency for Healthcare Research and Quality; 2019.

3. Moore BJ, Stocks C, Owens PL. Trends in Emergency Department visits, 2006-2014: Statistical Brief \#227. Rockville: Agency for Healthcare Research and Quality; 2017.

4. Zeller S. What psychiatrists need to know: patients in the Emergency Department. Psychiatr Times. 2018;35(8):1-4.

5. Nicks BA, Manthey DM. The Impact of psychiatric patient boarding in Emergency Departments. Emerg Med Int. 2012;2012:360308.

6. Freeman RE, Boggs KM, Zachrison KS, et al. National study of telepsychiatry use in U.S. Emergency Departments. Psychiatr Serv. 2020;71(6):540-6.

7. Credentialing Resource Center. Telepsychiatry continues to grow at a rapid rate. Medical Staff Briefing. 2018;28(7):1-4.

8. Hubley S, Lynch SB, Schneck C, Thomas M, Shore J. Review of key telepsychiatry outcomes. World J Psychiatry. 2016;6(2):269-82.

9. Thomas JF, Novins DK, Hosokawa PW, et al. The use of telepsychiatry to provide cost-efficient care during pediatric mental health emergencies. Psychiatr Serv. 2018;69(2):161-8.

10. Narasimhan M, Druss BG, Hockenberry JM, et al. Quality, utilization, and economic impact of a Statewide Emergency Department telepsychiatry program. Psychiatr Serv. 2015 Nov 1;66(11):1167.

11. Davies SF. A hospital driven telepsychiatry initiative to improve patient care and reduce costs. N C Med J. 2012;73(3):228-30.

12. Reliford A, Adebanjo B. Use of Telepsychiatry in pediatric emergency room to decrease length of stay for psychiatric patients, improve resident on-call burden, and reduce factors related to physician burnout. Telemed J E Health. 2019;25(9):828-32.

13. HCUP State Inpatient Databases (SID). Healthcare Cost and Utilization Project (HCUP). Rockville: Agency for Healthcare Research and Quality; 2005-2009. www.hcup-us.ahrq.gov/sidoverview.jsp. Accessed September 1, 2019.

14. HCUP State Emergency Department Databases (SEDD). Healthcare Cost and Utilization Project (HCUP). Rockville: Agency for Healthcare Research and Quality; 2009. www.hcup-us.ahrq.gov/seddo verview.jsp. Accessed September 1, 2019.

15. Barrett ML, Fingar KR, Owens PL, et al. Identifying observation services in the Healthcare Cost and Utilization Project (HCUP) State databases. 2015. HCUP Methods Series Report \#2015-05 ONLINE. U.S. Agency for Healthcare Research and Quality; 2015.

16. Sullivan AF, Richman IB, Ahn CJ, et al. A profile of US Emergency Departments in 2001. Ann Emerg Med. 2006;48:694-701.

17. Herscovici DM, Boggs KM, Sullivan AF, Camargo CA Jr. What is a freestanding Emergency Department? Definitions differ across major United States data sources. West J Emerg Med. 2020;21(3):660-4.

18. Zachrison KS, Boggs KM, Hayden E, et al. A national survey of telemedicine use by US Emergency Departments. J Telemed Telecare. 2020;26(5):278-84.

19. Core-Based Statistical Areas. Washington, DC: U.S. Census Bureau; 2016. https://www.census.gov/ topics/housing/housing-patterns/about/core-based-statistical-areas.html. Accessed December 1, 2019.

20. Critical Access Hospitals. Washington, DC: American hospital association; 2019. https://www.aha. org/advocacy/critical-access-hospitals. Accessed December 1, 2019. 
21. Association of American Medical Colleges. Council of Teaching Hospitals and Health Systems (COTH). AAMC; 2018. https://members.aamc.org/eweb/DynamicPage.aspx?site=AAMC\&webco de=AAMCOrgSearchResult\&orgtype=Hospital/Health\%20System. Accessed 1 Dec 2019.

22. American Hospital Association. Annual Survey dataset documentation manual. Chicago: American Hospital Association; 2016.

23. Boggs KM, Teferi MM, Espinola JA, Sullivan AF, Hasegawa K, Zachrison KS, et al. Consolidating Emergency Department-specific data to enable linkage with large administrative datasets. West J Emerg Med. 2020;21(6):141-5.

24. Owens PL, Fingar KR, McDermott KW, Muhuri PK, Heslin KC. Inpatient stays involving mental and substance use disorders, 2016. [Appendix B: ICD-10-CM codes defining mental and substance use disorders, by Category] Statistical Brief \#249. Rockville: Agency for Healthcare Research and Quality; 2019.

25. Bukhman AK, Baugh CW, Yun BJ. Alternative dispositions for Emergency Department patients. Emerg Med Clin North Am. 2020;38(3):647-61.

26. Zachrison KS, Boggs KM, Hayden EM, Espinola JA, Camargo JCA. Understanding barriers to telemedicine implementation in Rural Emergency Departments. Ann Emerg Med. 2020;75(3):392-9.

27. New York State Office of Mental Health. Office of Mental Health Appendix to United Statement Response to SED Report. https://omh.ny.gov/omhweb/resources/publications/sed-report.pdf. Accessed 15 Jan 2019.

28. McGinty KL, Saeed SA, Simmons SC, Yildirim Y. Telepsychiatry and E-Mental health services: potential for improving access to mental health care. Psychiatr Q. 2006;77(4):335-42.

29. New York State Office of Mental Health. Statewide comprehensive plan 2016-2020. https://omh. ny.gov/omhweb/planning/docs/507-plan.pdf. Accessed 1 Oct 2019.

30. Williams M, Pfeffer M, Boyle J, Hilty DM. Telepsychiatry in the Emergency Department: Overview and Case studies. Oakland, CA, California Healthcare Foundation. 2009. https://www.hasc.org/sites/ main/files/file-attachments/telepsychiatryprogramsed.pdf. Google Scholar.

31. Saeed SA. Tower of babel problem in telehealth: addressing the health information exchange needs of the North Carolina Statewide Telepsychiatry Program (NC-STeP). Psychiatr Q. 2018;89(2):489-95.

32. Maeng D, Richman JH, Lee HB, Hasselberg MJ. Impact of integrating psychiatric assessment officers via telepsychiatry on rural hospitals' emergency revisit rates. J Psychosom Res. 2020;133:109997.

33. Hilty DM, Cobb HC, Neufeld JD, Bourgeois JA, Yellowlees PM. Telepsychiatry reduces geographic physician disparity in rural settings, but is it financially feasible because of reimbursement? Psychiatr Clin North Am. 2008;31(1):85-94.

34. Centers for Medicare and Medicaid Services. Telehealth services [Internet]. Baltimore: CMS. 2016. https://www.cms.gov/Outreach-and-Education/Medicare-Learning-Network-MLN/MLNProducts/ Downloads/TelehealthSrvcsfctsht.pdf. Accessed 1 April 2020.

35. Centers for Medicare and Medicaid Services. Medicare program; revisions to payment policies under the physician fee schedule, clinical laboratory fee schedule, access to identifiable data for the center for medicare and medicaid innovation models and other revisions to part B for CY 2015. Final Rule with Comment Period. Fed Regist. 2014;72(219):67547-8010.

36. Mehrotra A, Huskamp HA, Souza J, et al. Rapid growth in mental health telemedicine use among rural medicare beneficiaries, wide variation across States. Health Aff. 2017;36(5):909-17.

37. Centers for Medicare and Medicaid Services. Medicare telemedicine health care provider fact sheets. CMS. 2020. https://www.cms.gov/newsroom/fact-sheets/medicare-telemedicine-health-care-providerfact-sheet. Accessed 1 April 2020.

38. Owens PL (AHRQ), Heslin KC (AHRQ), Fingar KR (IBM Watson Health), Weiss AJ (IBM Watson Health). Co-Occurrence of physical health conditions and mental health and substance use conditions among adult inpatient stays, 2010 Versus 2014. HCUP statistical brief \#240. Rockville: Agency for Healthcare Research and Quality; 2018. 
39. Hasegawa K, Brown DF, Tsugawa Y, Camargo CA Jr. Epidemiology of Emergency Department visits for opioid overdose: a population-based study. Mayo Clin Proc. 2014;89(4):462-71.

40. Goto T, Hasegawa K, Faridi MK, Sullivan AF, Camargo CA Jr. Emergency Department utilization by children in the USA, 2010-2011. West J Emerg Med. 2017;18(6):1042-6.

Publisher's Note Springer Nature remains neutral with regard to jurisdictional claims in published maps and institutional affiliations.

Cordelia Zhong is currently a Clinical Research Coordinator at the Emergency Medicine Network in the Department of Emergency Medicine at Massachusetts General Hospital.

Rain E. Freeman, MPH is an Epidemiology Specialist at the Center for Population Health Research at the University of Montana, Missoula.

Krislyn M. Boggs, MPH is a Senior Research Coordinator at the Emergency Medicine Network in the Department of Emergency Medicine at Massachusetts General Hospital.

Kori S. Zachrison, Md, MSc is an attending physician in the Department of Emergency Medicine at Massachusetts General Hospital. She also is an Associate Professor of Emergency Medicine at Harvard Medical School. Dr. Zachrison's academic focus is on the stroke systems of care and the role of telehealth in emergency care.

Jingya Gao, MS is a Biostatistician/Epidemiologist at the Emergency Medicine Network in the Department of Emergency Medicine at Massachusetts General Hospital.

Janice A. Espinola, MPH is a Biostatistician/Epidemiologist at the Emergency Medicine Network in the Department of Emergency Medicine at Massachusetts General Hospital.

Carlos A. Camargo, MD, DrPH is the Conn Chair in Emergency Medicine at Massachusetts General Hospital. $\mathrm{He}$ is also a Professor of Emergency Medicine and Medicine at Harvard Medical School, and Professor of Epidemiology at Harvard T.H. Chan School of Public Health. He is founder and director of the Emergency Medicine Network (EMNet), a multidisciplinary collaboration that aims to advance public health through diverse projects in emergency care, particularly multicenter clinical research. Dr. Camargo has an international reputation in respiratory/allergy emergencies, health services research in emergency care, health effects of vitamin D, and a variety of public health issues. He has served in many leadership roles, including President of the American College of Epidemiology. 